\title{
Síndrome de cordón umbilical corto
}

\author{
DOI 10.5377/alerta.v4i3.10956
}

Aurora Salazar de Escolero ${ }^{1^{*}}$, Nadia Verónica Fuentes Ruiz ${ }^{2}$, Alma Jeanette Handall Duron ${ }^{3}$, Zuleyma Yasmin Jaime Salvador ${ }^{4}$

1,2,3 y 4. Hospital San Juan de Dios de San Miguel, El Salvador

${ }^{*}$ Correspondencia

$\square$ aurorasalazarescolero@yahoo.com

1. (D) 0000-0002-7865-9046

\section{ACCESO ABIERTO}

\section{Body Stalk Syndrome}

\section{Citación recomendada:}

Salazar de Escolero A, Fuentes Ruiz NV, Handall Duron AJ, Jaime Salvador ZY. Síndrome de cordón umbilical corto. Alerta. 2021;4(3):98-102.

DOI 10.5377/alerta.v4i3.10956

\section{Recibido:}

3 de febrero de 2021

\section{Aceptado:}

8 de junio de 2021

\section{Publicado:}

26 de julio de 2021

\section{Contribución de autoría:}

ASdeE': Concepción y diseño del artículo, adquisición, análisis e interpretación bibliográfica, revisión crítica de contenido intelectual. NVFR ${ }^{2}, \mathrm{AJHD}^{3}$, ZYJS ${ }^{4}$ : Análisis e interpretación de información revisión crítica de contenido intelectual.

\section{Conflicto de intereses:}

Autoras declaran no tener conflictos de interés

\begin{abstract}
Resumen
El síndrome de cordón umbilical corto es una anomalía poco común e incompatible con la vida que se asocia a defectos de la pared anterior del feto, cordón umbilical corto o ausente y anomalías de los miembros. Esta entidad es la más severa y más infrecuente entre los defectos de la pared anterior del abdomen, con una incidencia de 1 en 14000 nacimientos. Se presenta el caso de un recién nacido de término, de sexo indeterminado, producto de primer embarazo, de parto abdominal. A las 28 semanas de gestación se diagnosticó un defecto en pared abdominal anterior y una imagen quística de columna vertebral por ultrasonografía. Al nacimiento se observaron los órganos abdominales expuestos, el cordón umbilical grueso, de $10 \mathrm{~cm}$ de longitud, eventración de intestinos e hígado y cifoescoliosis marcada, ausencia de genitales externos y de ano, un remanente de miembro inferior derecho y miembro inferior izquierdo completo con pie equinovaro. Se le dio apoyo ventilatorio y falleció a los 15 minutos de vida.

Palabras clave

Mortalidad perinatal, pared abdominal, anomalías congénitas, cordón umbilical.
\end{abstract}

\section{Abstract}

The abnormality of the development of the body stem constitutes an entity characterized by the presence of multiple rupture malformations, among which is a serious defect of closure of the abdominal wall with evisceration of the thoracoabdominal organs, severe kyphoscoliosis, absence of fusion between the chorion and the amnion, and short or absent umbilical cord. This entity is the most severe and rarest of the anterior abdominal wall defects, its incidence being 1 in 14000 births. The present case refers to a 22-year-old woman, a firth pregnancy from the rural area of Santa Rosa de Lima, La Unión, 28 weeks of gestational age, a fetus was found with a defect in the anterior abdominal wall plus a cystic image of the spine by means of ultrasound. Spontaneous labor begins at 37 weeks; cesarean section is performed for fetal malformations. Newborn with ambiguous genitalia and multiple malformations of the anterior abdominal wall, spine and lower limbs, confirmed the diagnosis of short umbilical cord syndrome, verified by the pediatrician who applies newborn care, dying after 15 minutes of life.

Keywords

Perinatal mortality, abdominal wall, congenital abnormalities, umbilical cord.

El desarrollo embriológico depende de dos factores, la genética y la epigenética, encargados de los caracteres hereditarios y específicamente del nivel de expresión de los genes en las células que pueden modificarse en situaciones ambientales desfavorables ${ }^{1}$

El síndrome de cordón umbilical corto, también conocido como limb-body wall complex y como síndrome body stalk, es una anomalía poco común e incompatible con la vida. Se trata de una malformación fenotípicamente variable que suele asociar defectos de la pared anterior del feto, cordón umbilical corto o ausente y anomalías de los miembros². El cordón umbilical, que es la conexión entre madre e hijo, juega un importante papel en esta relación². La longitud y enrollamiento de este cordón son índices de movilidad fetal y, por el contrario, el cordón umbilical corto se relaciona con malformaciones fetales especialmente con defectos de pared abdominal, extremidades y columna vertebral ${ }^{3}$. Además, presentan bajo coeficiente intelectual y pobre desarrollo embriológico ${ }^{2-5}$. 
El de cordón umbilical corto se presenta en uno de cada 10000 a 40000 embarazos $^{3,4}$. Su etiología es controvertida y no existe evidencia de causa genética. Afecta por igual a ambos sexos y no se ha encontrado asociación con técnicas de reproducción asistida ${ }^{2-5}$.

La única forma de detectar oportunamente esta anomalía es por medio de ultrasonografía y debe considerarse esta patología ante un defecto severo de la pared abdominal ${ }^{3}$. Además, en la mayoría de los casos se observa un aumento de la translucencia nucal y la elevación de la alfafetoproteína ${ }^{3}$, de ahí la importancia de seguir adecuadamente el protocolo de ultrasonografías durante el control prenatal $\left.\right|^{6-11}$, donde la primera se indica de las 11 a las 13 semanas 6 días, la segunda de las 18 a las 24 semanas y la última en el final del tercer trimestre ${ }^{6}$.

El control prenatal adecuado proporciona beneficios para el diagnóstico prenatal y genera objetivos concretos en torno al manejo de estos casos. Determina si el recién nacido será compatible con la vida, la consejería y el manejo psicológico de la madre y la preparación de un equipo multidisciplinario para la atención prenatal y del parto 6 .

\section{Presentación del caso}

Recién nacido de término, de sexo indeterminado, producto de primer embarazo. La madre, de 22 años, inició los controles prenatales a las 12 semanas de gestación en la unidad comunitaria, sin comórbidos, ni consumo sustancias nocivas, con resultado de tipeo y Rh O positivo, VIH y VDRL negativos. Se practicó una ultrasonografía prenatal a las 28 semanas de gestación en la que se observó un feto único con defecto en pared abdominal anterior más imagen quística de columna vertebral, fue referido al Hospital Nacional de la Mujer para realizar el estudio, pero este no se completó.

El 4 de agosto del 2020, consultó en la unidad de emergencia del Hospital San Juan de Dios de San Miguel, con embarazo de 37 semanas e inicio de trabajo de parto espontáneo; se indicó parto vía abdominal por el diagnóstico de una patología fetal y nació un producto vivo, cefálico, con abundante líquido amniótico meconial y con esfuerzo respiratorio débil. Al examen físico se observaron los órganos abdominales expuestos, recubiertos por una membrana adherida al cordón umbilical, este era grueso de $10 \mathrm{~cm}$ de longitud y con abundante gelatina de Wharton. Además, presentaba eventración de intestinos e hígado (Figura 1), con cifoescoliosis marcada y ausencia de genitales externos y ano (Figura 2). A ni- vel de extremidades inferiores se observó un remanente de miembro inferior derecho y miembro inferior izquierdo completo con pie equinovaro. El recién nacido presentó $1760 \mathrm{~g}$ de peso, talla de $36 \mathrm{~cm}$, perímetro cefálico de $31 \mathrm{~cm}$, perímetro torácico de 23 $\mathrm{cm}$, perímetro abdominal de $20 \mathrm{~cm}$, Apgar de 5 al primer minuto y 6 a los 5 minutos, edad gestacional por Ballard para 37 semanas y no se observaron anomalías craneofaciales ni torácicas (Figura 3). Se colocó en una cuna térmica, donde se le aspiraron las secreciones, se secó y se le cortó el cordón umbilical. Se intubó, como apoyo ventilatorio, fue evaluado por el neonatólogo y el cirujano pediatra, quienes refirieron que la anomalía era invariablemente mortal. El neonato falleció a los 15 minutos de vida.

\section{Discusión}

El síndrome de cordón umbilical corto es una malformación congénita incompatible con la vida, que se caracteriza por la presencia de defecto mayor de pared abdominal anterior, el cordón umbilical corto o ausente, la continuidad entre la pared abdominal y la placenta, cifoescoliosis, malformaciones craneofaciales (encefalocele, fisura labiopalatina) y de las extremidades ${ }^{12-14}$.

No existe una clasificación que cubra todo el espectro de defectos de la pared abdominal anterior ${ }^{7}$.

Estos defectos se clasifican, de forma práctica, como periumbilicales (onfalocele y gastrosquisis), de la línea media inferior (extrofia de vejiga y extrofia de cloaca) y de la línea media superior (pentalogía de Cantrell) $)^{7}$. Otras malformaciones menos frecuentes de la pared abdominal anterior son la hernia epigástrica congénita, la displasia de pared corporal, el síndrome de Prune Belly o síndrome de vientre en ciruela pasa, la hendidura epigástrica, los defectos de pared asociados al síndrome de bridas amnióticas (síndrome de cordón corto) ${ }^{8-11}$.

El origen embriológico de estas malformaciones viene desde el día 21 al 28, período en el cual se producen cuatro plegamientos en el disco germinal: cefálico, caudal y dos laterales ${ }^{15,16}$. Se forma un tallo en la porción ventral que formará el cordón umbilical, que en ese momento se encuentra conectado a la placenta, el cual formará parte del cordón umbilical después del plegado del disco embrionario ${ }^{16}$.

La cavidad amniótica crece y rodea al embrión obliterando la cavidad coriónica y el amnios se fusiona con el corion a nivel del cordón umbilical ${ }^{2,16}$. Cuando el plegado no se produce adecuadamente, aparecen malformaciones graves ${ }^{2,16}$. 


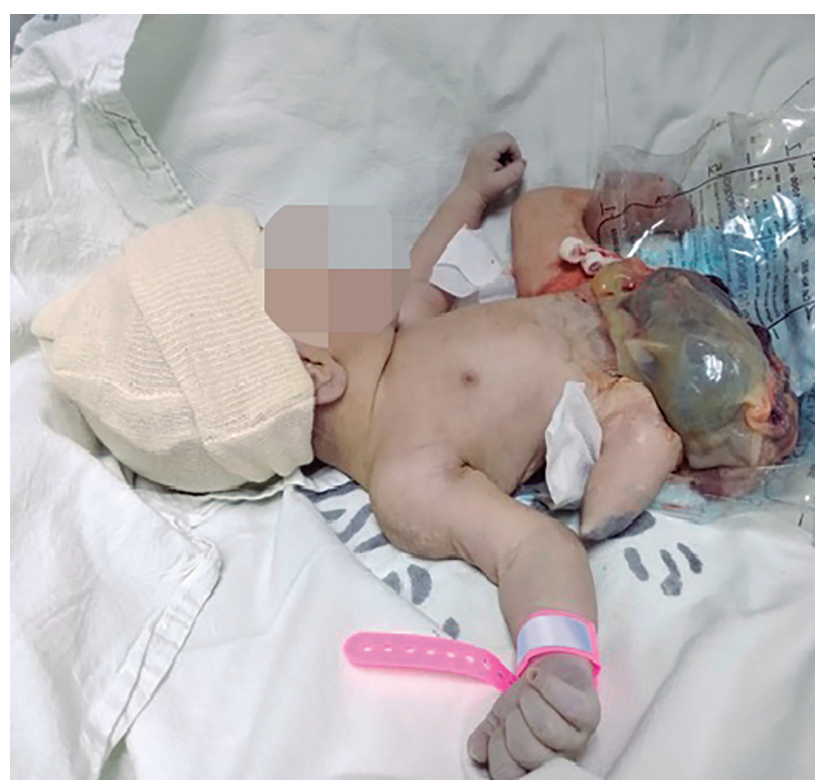

Figura 1. Recién nacido con síndrome de body stalk en el primer minuto de vida donde se observan características propias del síndrome.

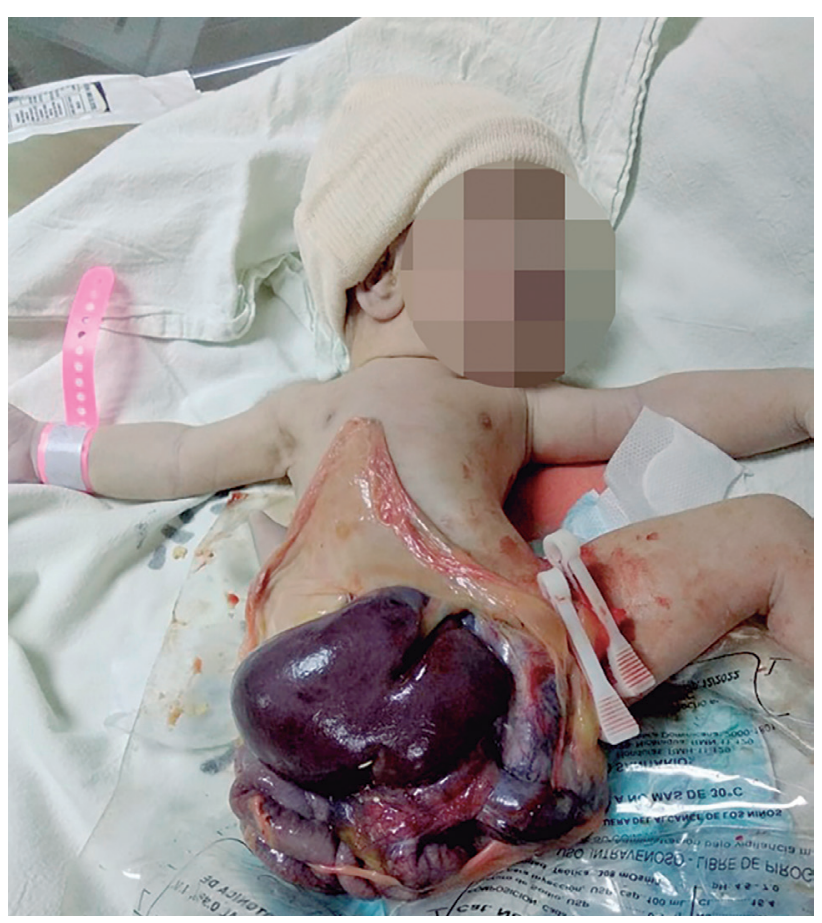

Figura 2. Recién nacido con síndrome de body stalk (exposición de vísceras abdominales, ausencia de genitales externos y miembro inferior derecho).

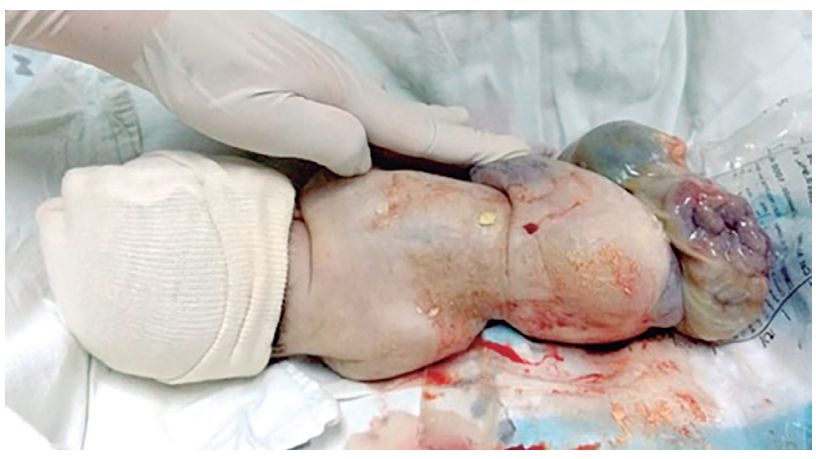

Figura 3. Recién nacido con cifoescoliosis
Existen algunas hipótesis que han querido explicar la causa primaria de este síndrome. La teoría más clásica propone que una ruptura muy temprana de las membranas amnióticas causa traspaso de líquido amniótico a la cavidad celómica; como consecuencia, las partes fetales escapan a esta cavidad, tras reabsorberse el líquido y se forman constricciones, amputaciones y otras malformaciones en un fenómeno similar al síndrome de bandas amnióticas².

Otra hipótesis sugiere una interrupción en la vascularización fetal, a través de las arterias uterinas o determinados teratógenos, que conduce a una necrosis hemorrágica fetal con la consiguiente pérdida de tejidos, interrupción en el desarrollo y generación de adherencias ${ }^{5,15-18}$.

En relación al diagnóstico, la alfafetoproteína plasmática materna puede estar normal o aumentada, a diferencia del cariotipo, que suele ser normal, por lo que su realización rutinaria no está indicada.

Los hallazgos ultrasonográficos, desde las 11 semanas de embarazo, son: cordón umbilical rudimentario o ausente, feto fusionado con placenta, movilidad disminuida, defecto pared toraco-abdominal, malformaciones en extremidades, partes fetales en cavidad celómica.

Por otro lado, resulta sumamente importante distinguir esta malformación de otros defectos de pared anterior, sobre todo de aquellos que puedan corregirse y sean compatibles con la vida ${ }^{3,5,7}$. Entre los diagnósticos diferenciales se menciona el onfalocele, que es un defecto congénito de la pared abdominal anterior, a través del cual se hernia el contenido abdominal, cubierto por una membrana de tres capas (peritoneo, gelatina de Wharton y amnios) ${ }^{28,16}$; la gastrosquisis que se describe como un defecto congénito de todas las capas de la pared abdominal anterior, localizado a un lado del cordón umbilical normal (en general del lado derecho), a través del cual protruye el contenido abdominal, desprovisto de cualquier tipo de envoltura ${ }^{2,8,16}$; la hernia umbilical que se presenta en la pared abdominal intacta con asas intestinales situadas en el interior del cordón umbilical2,16; la pentalogía de Cantrell, también conocida como síndrome de Cantrell-Haller-Ravitch o hernia diafragmática pericárdica-peritoneal, que se caracteriza por defectos de la pared anterior en la línea media epigástrica, de la parte inferior del esternón, del diafragma anterior, del pericardio diafragmático y otros defectos cardíacos ${ }^{2,8}$; el síndrome de Beckwith-Wiedeman, que incluye onfalocele, macroglosia y organomegalia 2,16,20; la extrofia vesical, en la que se observa una pared anterior de vejiga ausente 
y como consecuencia es la pared posterior la que se halla evertida y expuesta ${ }^{2,8}$; la extrofia cloacal, una anomalía compleja y poco frecuente que involucra al tracto urinario e intestinal debido a un defecto en la formación del septum urorectal ${ }^{2,8}$, y el síndrome de banda amniótica, también conocido como complejo de ADAM, un complejo malformativo que puede presentar desde pequeños anillos de constricción y linfedema en los dedos, hasta severas anomalías atribuidas a la presencia de bandas amnióticas que comprimen y amputan diversas partes del cuer$\mathrm{po}^{2}$.

En el caso del síndrome de cordón umbilical corto, su diagnóstico debe hacerse lo más temprano posible, idealmente en el primer trimestre de gestación, para poder dar atención psicológica a la paciente y su familia; además, si las condiciones obstétricas lo permiten, elegir la vía del parto vaginal, debido a que el feto no tiene oportunidad de vida extrauterina y disminuye el riesgo de complicaciones maternas ${ }^{2,10}$. En este caso, se decidió la vía abdominal debido a que no se contaba con la confirmación del diagnóstico prenatal.

En estos casos se recomienda el estudio con radiografías y prueba de cariotipo; sinembargo, estos no estuvieron disponibles en la atención prenatal.

El diagnóstico definitivo y temprano es importante para el abordaje psicológico y la elección de la vía del parto. La interrupción voluntaria del embarazo en los casos diagnosticados con una patología fetal que no tiene pronóstico de vida se realiza en los países donde la legislación permite el aborto eugénico 14,16,19,20.

\section{Conclusión}

El diagnóstico del síndrome de cordón umbilical corto en el período prenatal y de manera oportuna es importante debido al pronóstico fetal, que permite brindar una adecuada consejería y manejo psicológico a la madre y preparar un equipo multidisciplinario para la atención prenatal y del parto.

\section{Agradecimiento}

A la Dra. Marjorie Galeano y la Dra. Carolina Mena, por su ayuda en la identificación de la malformación.

\section{Financiamiento}

Autoras declaran no tener fuente de financiamiento.

\section{Referencias bibliográficas}

1. Pietrantuono Ml. Epigenética y desarrollo embrionario: cambios con consecuencias. Consejo Nacional de Investigaciones Científicas y Técnicas. 2014. Fecha de consulta: 28 de enero de 2021. Disponible en: https:// bit.ly/3pHnATx

2. Díaz O, Hurtado Sánchez M, Carrillo B. Diagnostico ecográfico del síndrome de Body Stalk. Rev. Latin. Perinat. 2018.21(3):172-76. Disponible en: http://www.revperinatologia. com/images/10 Body Stalk.pdf

3. Revels JW, Wang SS, Nasrullah A, Revzin M, lyer RS, Deutsch G, et al. An algorithmic approach to complex fetal abdominal wall defects. AJR Am J Roentgenol. 2020;214(1):218-231. DOI: 10.2214/AJR.19.21627

4. Santiago M, Sarmiento C, Loyo J, Rivera L, Cabrera Lozada C. Diagnóstico ecográfico fetal de defectos complejos de pared abdominal. Rev. Latin.Perinat.2017;20(4):258-262. Disponible en: https://bit.ly/3vg34dU

5. Díaz C, Copado Y, Muñoz G, Muñoz H. Malformaciones de la pared abdominal. Rev. Med. Clin. Condes. 2016;27(4):499-508. DOl:10.1016/j.rmclc.2016.07.009

6. Ministerio de Salud. Lineamientos técnicos para la atención de la mujer en el período preconcepcional, prenatal, parto, puerperio y al recién nacido. San Salvador. Ministerio de Salud. 2021. 214 p. Disponible en: https://bit. ly/3x932pz

7. Cafici D, Sepulveda W. Evaluacion ultrasonografica de los defectos de cierre de la pared anterior del abdomen fetal. 1a edición. Buenos Aires. Ediciones Journal; 2007. Capítulo 15, Defectos del cierre de la pared anterior del abdomen. Pg 321- 342.

8. Victoria T, Andronikou S, Bowen D, Laje P, Weiss DA, Johnson AM, et al. Fetal anterior abdominal wall defects: prenatal imaging by magnetic resonance imaging. Pediatr Radiol. 2018;48(4):499-512. DOI: 10.1007/s00247-0173914-x

9. Coleman PW, Marine MB, Weida JN, Gray BW, Billmire DF, Brown BP. Fetal MRI in the identification of a fetal ventral wall defect spectrum. AJP Rep. 2018;8(4):264-276. DOI: 10.1055/s-0038-1675353

10. Núñez-Sánchez GC, Gallardo-Gaona JM, Velázquez-Torres B, Camarena-Cabrera DM, Acevedo-Gallegos S, RamírezCalvo JA. Rendimiento diagnóstico del ultrasonido del primer trimestre para alteraciones estructurales. Ginecol Obstet Mex. 2021;89(1):5-13. DOI: 10.24245/gom. v89i1.3540

11. Delgado Montano FJ, Mena Ugarte SC. Síndrome de banda amniótica, un diagnóstico 
a través de imágenes. Revista ALERTA. 2019; 2(2). DOI: 10.5377/alerta.v2i2.7921

12. Pardo Vargas RA, Aracena M, Aravena T, Cares C, Cortés F, Faundes V, et al. Consenso de la Rama de Genética de la Sociedad Chilena de Pediatría sobre las anomalías congénitas de mal pronóstico vital (ACMPV): Genetics Consensus Committee. Rev chil pediatr. 2016;87(5):422-431. DOI: 10.1016/j. rchipe.2016.04.005

13. Putti P. Defectos congénitos y patologías incompatibles con la vida extrauterina. Rev Med Urug. 2016;32(3):218-223. Disponible en: https://bit.ly/3izqdoT

14. Rossini MM, Nuñes de Faria Stamm AM. Malformación fetal incompatible con la vida: conducta de neonatólogos. Rev Bioét. 2020;28(3):531-536. DOI: 10.1590/198380422020283417

15. Marfull $\mathrm{C}$, Illanes $\mathrm{S}$, Figueroa $\mathrm{H}$, Yamamoto M. Actualización: banda amniótica y terapia fetal. Rev Chil Ultrasonog. 2019;22(1): 06-16. Disponible en: https://bit.ly/3zggadr

16. Martínez Leyva G, Blanco Pereira ME, Rodríguez Acosta Y, Enríquez Domínguez L, Marrero Delgado I. De la embriogénesis a la prevención de cardiopatías congénitas, defectos del tubo neural y de pared abdominal. Rev Méd Elect. 2016;38(2):239-50. Disponible en: https://bit.ly/3iBzDjO

17. Diaz Primera RJ, Gil Guevara ED, Sánchez Jiménez R, Bermúdez González C. Cirugía fetal en bandas amnióticas. Rev. Peru Ginecol Obstet. 2018;64(4):639-645. DOI: 10.31403/ rpgo.v64i2134

18. Ferrer Montoya R, Hernández Reyes A, Ortiz Pérez G. Síndrome de Bridas Amnióticas. Revisión Bibliográfica. Multimed. 2016;20(5): 251-259 Disponible en: http://www. revmultimed.sld.cu/index.php/mtm/article/ view/404

19. Vitaliano Vadillo AA. ¿Por qué se debe despenalizar el aborto eugenésico en la legislación peruana? NOUS, Revista de Investigación Jurídica de Estudiantes. 2016;7(9):85-103. Disponible en: https://bit. ly/3gtZp7b

20. Fernández Ladrón V, Arrieta Bretón S, Sáenz Pascual MP, San Martín Basterra C, Lorente Álava M. Gestación de 28 semanas con anomalía del tallo corporal. Prog Obstet Ginecol 2018;61(6):582-585 Disponible en: https://bit.ly/3izNaZg 